\title{
Quality by design approach to protein PEGylation: a review
}

\author{
Smriti Rajan Patel*, Debjani Dasgupta \\ School of Biotechnology and Bioinformatics, D. Y. Patil University, Navi Mumbai, Maharashtra, India.
}

\section{ARTICLE INFO}

Article history:

Received on: 14/06/2017

Accepted on: 26/07/2017

Available online: 14/08/2017

\section{Key words:}

Polyethylene glycol (PEG), quality by design (QbD), critical quality attributes (CQAs), critical process parameters (CPPs), design of experiments (DOE) and design space.

\begin{abstract}
The Food and Drug Administration (FDA) first approved PEGylated product in 1990, since then PEGylation, as a modification procedure for enhancing biomedical efficiency and physicochemical properties of therapeutic proteins has been extensively used. Recombinant proteins are prone to rapid degradation due to proteolysis or may have a brief circulating half-life due to low renal filtration. These limitations can be overcome by PEGylation, where polyethylene glycol chains are linked to peptides and protein molecules. The quality by design $(\mathrm{QbD})$ paradigm helps to develop a process design spaces which describes the interactions and multidimensional effects of method variables on critical quality attributes of therapeutic proteins. The complexities involved in manufacturing processes have led to the development of strategies to establish a design space, ensuring reliable and reproducible outcomes. QbD approach in process optimization allows simultaneous screening of process variables, thus reducing the number of tests conducted as compared to the traditional approach based on a trial and error method. An approach to QbD using the design of experiments (DOE) has been used to establish a design space for PEGylation of recombinant proteins. The aim of this paper is to provide a systematic approach for implementing quality by design for development of a protein PEGylation process.
\end{abstract}

\section{INTRODUCTION}

Polyethylene glycol (PEG) is a synthetic polymer consisting of repeating units of ethylene oxide. Being nonimmunogenic, non-antigenic, nontoxic and amphiphilic in nature, PEG FDA has approved the use of PEG in human oral, intravenous, and dermal pharmaceutical preparations. Due to these properties, PEG as a modifying polymer plays a vital role in drug delivery. The concept of PEGylation for enhancing the therapeutic and biotechnological potential of peptides and proteins is widely used. Linking of polyethylene glycol to a polypeptide can alter many of its features without affecting its main biological functions, such as enzymatic activity or receptor recognition. PEG conjugation to a protein disguises its surface and increases the molecular weight of the polypeptide, thus reducing its renal filtration, immunogenicity and susceptibility towards proteolytic cleavage. PEGylation is therefore of interest

* Corresponding Author Smriti Rajan Patel, Smriti Rajan Patel (PhD student), School of Biotechnology and Bioinformatics, D. Y. Patil University, Level 5, Sector 15, Plot No. 50, CBD Belapur, Navi Mumbai, Maharashtra 400614, India.Email: smritipatel86 @ gmail.com, Phone: +919619154055 in applied biotechnology. Details of FDA approved PEGylated proteins, antibody fragments and oligonucleotides are listed in table1 [1, 2]. The Biopharmaceutical industry is rigorously exploring for means to ensure and improve product safety, quality and efficacy. Conversely, drug recalls, manufacturing failure cost, scale up obstacles and regulatory problem in recent past imply otherwise. Using the conventional quality by testing $(\mathrm{QbT})$ method to testing the final product for product quality and performance leading to the inadequate knowledge of the process and critical process parameters. Regulatory authorities ensure the implementation of quality by design $(\mathrm{QbD})$, which is a scientific methodology to enhance process understanding by reducing the process discrepancies and enabling process control strategies. Table 2 highlights the differences when using a traditional approach versus the $\mathrm{QbD}$ approach during product development $[3,4]$. QbD approach mentioned in the ICH guidelines Q8, Q9 and Q11 [5-7] emphasizes the requirement of in-depth process knowledge to achieve consistent product quality along with a robust manufacturing process and not just product qualification. The key tools of $\mathrm{QbD}$ are; incorporation of prior knowledge, the use of statistically designed experiments, risk and knowledge management. Implementation of $\mathrm{QbD}$ requires in-depth information regarding product and its manufacturing process. 
Table 1: FDA approved PEGylated drugs [2].

\begin{tabular}{ccccc}
\hline Commercial name & Drug name & Company & PEG size (Da) & Year of \\
approval
\end{tabular}

Abbreviations: ALL: acute lymphoblastic leukemia; CML: chronic myeloid leukemia; GH: growth hormone; SCID: severe combined immunodeficiency disease

Table 2: Difference between current approach and QbD approach [11].

\begin{tabular}{ll}
\hline \multicolumn{1}{c}{ Traditional Approach } & \multicolumn{1}{c}{ QbD Approach } \\
\hline $\begin{array}{l}\text { Quality is assured by testing and inspection. } \\
\begin{array}{l}\text { It includes only data intensive submission which includes } \\
\text { disjointed information without "big picture". }\end{array}\end{array}$ & $\begin{array}{l}\text { Quality is built into product \& process by design and based on scientific understanding. } \\
\text { understanding. }\end{array}$ \\
\hline $\begin{array}{l}\text { Here, any specifications are based on batch history. } \\
\text { Here there is "Frozen process," which always discourages changes. }\end{array}$ & Here, any specifications based on product performance requirements. \\
$\begin{array}{l}\text { It focuses on reproducibility which often avoids or ignores } \\
\text { variation. }\end{array}$ & It focuses on robustness which understands and control variation. \\
\hline
\end{tabular}

Table 3: Examples of CQAs \& CPPs for the unit process of PEGylation.

\begin{tabular}{ccc}
\hline Unit process & Critical Quality Attributes (CQAs) & Critical Process Parameters (CPPs) \\
\hline \multirow{3}{*}{ PEGylation } & Visual attributes & PEG: Protein ratio \\
& Appearance & Reaction $\mathrm{pH}$ \\
& Dissolution & Reaction temperature \\
Percentage conversion & Reaction time \\
& Yield & Purity of the PEG moiety \\
\hline
\end{tabular}

It is not feasible to study the effect of every variable on thesafety and efficacy of a product due to the interactions between the large number of variables and attributes, hence statistical tools such as design of experiments (DOE) along with risk assessment like the failure modes and effects and analysis (FMEA), help to ensure that resources are spent towards the important processes. Efficacious implementation of QbD requires detailed knowledge and understanding of the interactions between the critical quality attributes (CQAs) and the clinical properties of the product. It also includes an understanding of the association between the process and CQAs. Experimental design and analysis help in understanding the influence of critical process parameters (CPPs) on CQAs and therefore can identify and control the sources of variability $[4,8-10]$.

\section{IMPLEMENTATION OF QbD FOR THE UNIT PROCESS OF PEGYLATION}

Development of new biopharmaceutical product using the $\mathrm{QbD}$ approach can go through the following steps;

\subsection{Quality target product profile (QTPP)}

QTPP summarizes the drug product quality characteristics to be achieved in terms of safety and efficacy.
For PEGylation, percentage purity and the stability of the PEGylated protein can be considered as the QTPP.

\subsection{Determining critical process parameters (CPPs) and critical quality attributes (CQAs)}

CPPs are parameters whose inconsistencies would impact CQAs and should be controlled to assure that the method consistently produces the desired quality in terms of percentage purity and yield of the PEGylated protein [12]. CQAs are physical, chemical, biological, or microbiological attributes that can be measured and monitored to ensure the desired product output remains within the acceptable quality limits [13]. As seen in table 3, parameters responsible for efficient PEGylation process are segregated as CQAs and CPPs. For example, the quality of the PEG moiety determines the quality of the conjugate formed post PEGylation.

The structure and quality of the final PEGylated product would depend on factors like; the presence of impurities, the molecular weight (MW) distribution of the polymer, and reactive group chemistry [14]. Thus, the stability and purity of the PEG reagent are the CPPs and the outcome, i.e. percentage conversion and yield of the PEGylated protein are the CQAs. The CPPs and CQAs for the PEGylation process can be identified as per risk assessment. 


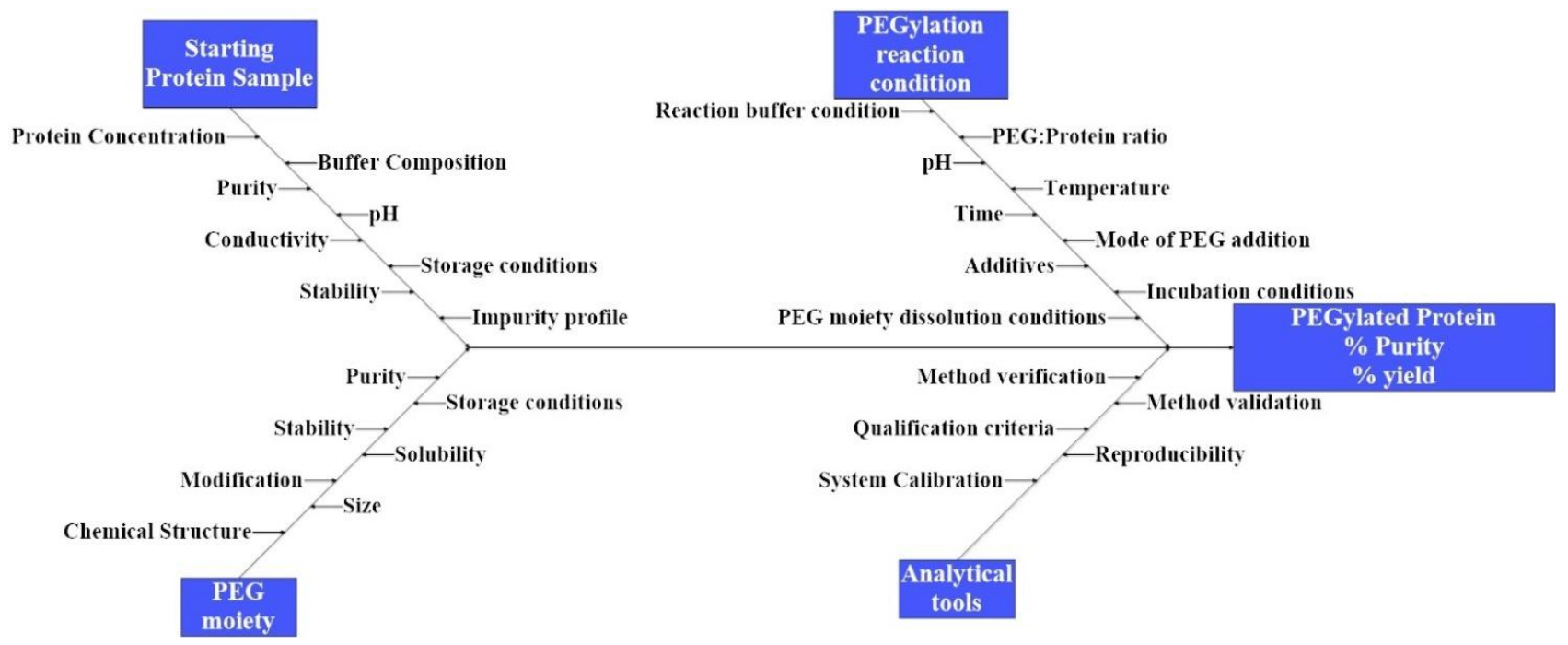

Fig. 1: Ishikawa diagram of protein PEGylation compression process.

\subsection{Risk assessment}

Risk assessment aims to obtain all the potentially high impact factor parameters which will be subjected to a DOE study to establish a process design space. Risk assessment includes tools like the Ishikawa (cause and effect) diagram, failure mode effect analysis (FMEA), and pareto plot. An Ishikawa diagram helps detect all probable variables which could have an impact on a CQA. Failure modes and effect analysis (FMEA) is used to assess the level of risk for each processing step in an orderly manner and to prioritize experiments to be evaluate based on their impact on the process outcome. Figure 1 shows the FMEA process of risk assessment. In risk assessment, variables are ranked based on the combination of occurrence $(\mathrm{O})$, severity $(\mathrm{S})$ and detectability (D). $\mathrm{O}$ is the occurrence possibility of an incident occurring. The next parameter S (severity) measures the criticality of a given effect that could hamper the process/product quality. The ease at which a failure mode can be detected defines the parameter D. The more measurable a failure mode is, the lesser risk process holds. Multiplying these values would result in the risk priority number (RPN) [15].

The different failure modes identified for the PEGylation process along with their RPN scores are plotted against each other to identify the failure modes that are given highest importance during the experimentations. This forms the pareto plot. The selection or omission of process parameters for a study is justified through pare to plots [16]. Through risk assessment, parameters with high risk parameters are further studied to understand their effects on CQAs/CPPs.

\subsection{Developing a design space}

ICH guideline Q8 (R2) defines design space as a multidimensional combination of input variables (e.g., material attributes) and their interactions with the process parameters to provide assured quality product [5]. Design of experiments (DOE) help establish a design space can be established for a unit process, multiple unit operations, or for the complete production process. DOE is a controlled, structured method which determines the relationships between the factors affecting a process and its outcome. Various mathematical DOE models like PlacketBurman, Full and fractional factorial designs, Surface design, BoxBehnken and Response surface methodology (RSM) are available [17]. Studies are designed using DOE; such that the data obtained is used in understanding and defining the design space. Benefits of using QbD are as follows;

- In-depth information can be obtained by conducting a minimum number of experiments.

- $\quad$ Effects of individual parameters can be studied simultaneously by altering all the operating parameters.

- DOE accounts for discrepancies in experiments, materials, process or operators.

- In-sight into the interaction between different variables can be achieved.

- Helps to determine a design space through identified acceptable ranges of critical process parameters.

Based on prior knowledge and risk assessment, the parameters having a high RPN score are selected and are included in the multivariate study of DOE. The selection of an appropriate experimental design depends on the objective of the study (e.g., initial screening, optimization, or robustness study), the CQAs/CPPs and their interactions with the process and available resources such as literature knowledge, raw materials, labor time and cost. The level of the factor range to be set depends on the purpose of the experiment. Screening experiments involve the use of full or fractional designs where the variables are studied over a wide range to increase the likelihood of covering the optimum conditions and to attain desired effects. 

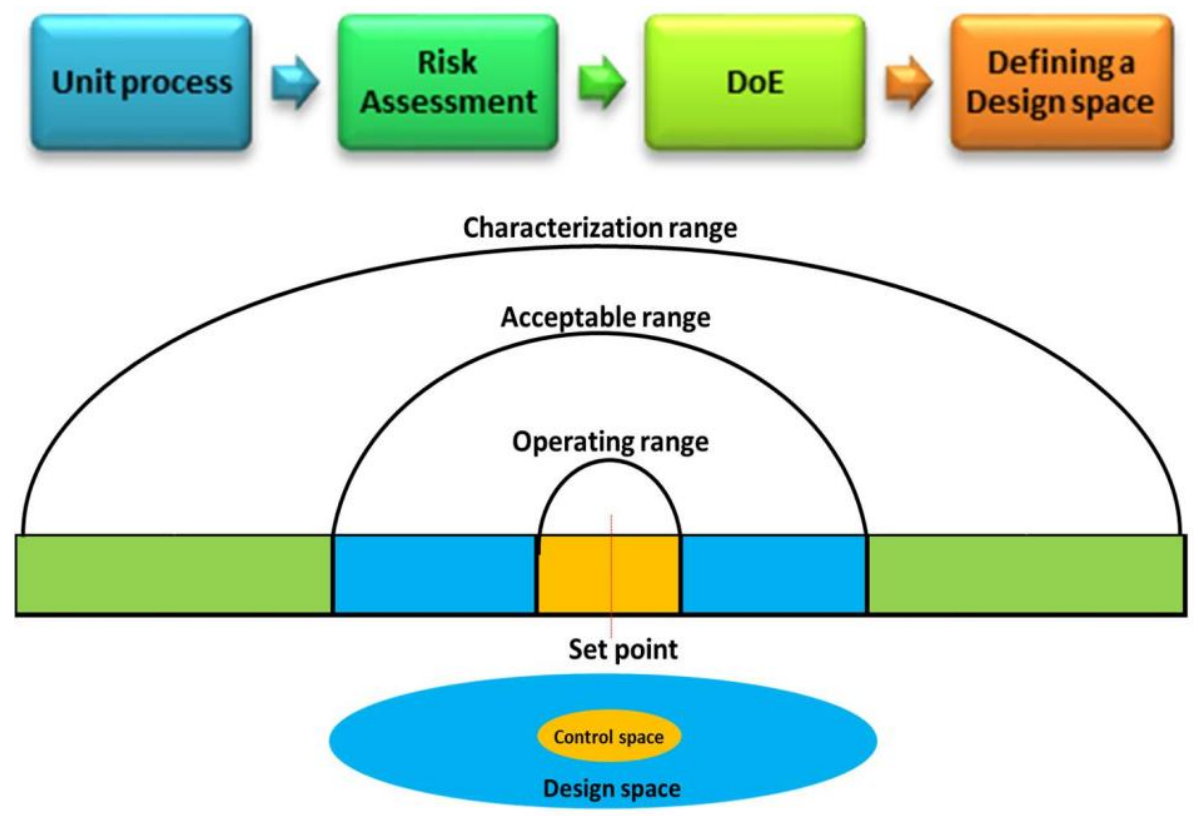

Fig. 2: Process design space.

Broader the range more stable is the model. In optimizing studies, the range should narrow, as more information regarding variables and their implications are available at this stage. The results obtained are analyzed to identify the important parameters and their role in establishing a design space. When DOE is applied to the PEGylation process, factors are the process parameters (e.g., PEG to protein ratio, $\mathrm{pH}$, target protein concentration and time), while responses are the critical quality attributes such as yield, percentage purity and percentage conversion of the target protein to its PEGylated form. A result obtained from the DOE output aid in identifying the most favorable conditions, the crucial factors that influence CQAs and the less impacting ones and provides detailed information regarding the interactions and synergies between factors. Figure 2 depicts the creation of a design space from results obtained during process characterization studies and its association with the characterization and operating space. The operating ranges defined by the unit process modus operandi comprise the operating space, while the process characterization work is conducted within the characterization space. The process design space is defined as an acceptable range which is an outcome of the product characterization studies [18].

\subsection{Defining a control strategy}

A control strategy is designed to ensure consistent production of quality product.Control strategy is based on a defined set of controls, resultant of product and process understanding that ensures consistent process performance and product quality. The control strategy a subset of the QbD model is established via risk assessment that considers the criticality of the CQAs and CPPs. Control space should lay within the design space [19].

\section{CONCLUSION}

The FDA, European and Japanese regulatory agencies are stressing on the implementation of $\mathrm{QbD}$ which aims to produce a consistent and high-quality biopharmaceutical product through a rapid and a robust method. This article demonstrates the benefits of applying QbD principles towards process optimization for PEGylation of proteins, instead of using the traditional approach. With the QbD approach, the product quality is not only tested at the end of the manufacturing process, but is incorporated at every step involved in product manufacturing. Quality assurance is a superior to quality control and in $\mathrm{QbD}$; quality is assured rather than controlled which is of significance during development of biotech therapeutics.

\section{Financial support and sponsorship: Nil.}

Conflict of Interests: There are no conflicts of interest.

\section{REFERENCES}

1. Veronese F. Peptide and protein PEGylation. Biomaterials. 2001; 22(5):405-417.

2. Li W, Zhan P, De Clercq E, Lou H, Liu X. Current drug research on PEGylation with small molecular agents. Progress in Polymer Science. 2013; 38:421-444.

3. Jain $\mathrm{S}$. Quality by Design $(\mathrm{QbD})$ : A comprehensive understanding of implementation and challenges in pharmaceuticals development. International Journal of Pharmacy and Pharmaceutical Sciences. 2014; 6(1):29-35.

4. Chang R, Raw A, Lionberger R, Yu L. Generic development of topical dermatologic products, Part II: Quality by design for topical semisolid products. The AAPS Journal. 2013; 15(3):674-683.

5. ICH Harmonised tripartite guideline for pharmaceutical development Q8 (R2). 2009. Available from: https://www.ich.org/fileadmin/Public_Web_Site/ICH_Products/Guid elines/Quality/Q8_R1/Step4/Q8_R2_Guideline.pdf. 
6. ICH Harmonised tripartite guideline for pharmaceutical development Q9. 2005. Available from: https://www.ich.org/fileadmin/Public_Web_Site/ICH_Products/Guid elines/Quality/Q9/Step4/Q9_Guideline.pdf.

7. ICH Harmonised tripartite guideline for pharmaceutical development Q10. 2005. Available from: https://www.ich.org/fileadmin/Public_Web_Site/ICH_Products/Guid elines/Quality/Q10/Step4/Q10_Guideline.pdf.

8. Meitz A, Sagmeister P, Langemann T, Herwig C. An integrated downstream process development strategy along $\mathrm{QbD}$ principles. Bioengineering. 2014; 1(4):213-230.

9. Elliott $\mathrm{P}$, Billingham $\mathrm{S}$, Bi J, Zhang $\mathrm{H}$. Quality by design for biopharmaceuticals: a historical review and guide for implementation. Pharmaceutical Bioprocessing. 2013; 1(1):105-122.

10. Rathore A, Pathak M, Godara A. Process development in the QbD paradigm: role of process integration in process optimization for production of biotherapeutics. Biotechnology Progress. 2015; 32(2):355-362.

11. Patil A, Pethe A. Quality by Design (QbD): A new concept for development of quality pharmaceuticals. International Journal of Pharmaceutical Quality Assurance. 2013; 4(2):13-19.

12. Boussès C, Ferey L, Vedrines E, Gaudin K. Using an innovative combination of quality-by-design and green analytical chemistry approaches for the development of a stability indicating UHPLC method in pharmaceutical products. Journal of Pharmaceutical and Biomedical Analysis. 2015; 115:114-122.

13. Nagar M, Singhai S, Chopra V, Bala I, Trivedi P. A study over effects of process parameters on quality attributes of a tablet by applying "quality by design". Der Pharmacia Lettre. 2010; 2(2): 370392.
14. Payne R, Murphy B, Manning M. Product development issues for PEGylated proteins. Pharmaceutical Development and Technology. 2010; 16(5):423-440.

15. Fahmy R, Kona R, Dandu R, Xie W, Claycamp G, Hoag S. Quality by design I: Application of Failure Mode Effect Analysis (FMEA) and Plackett-Burman design of experiments in the identification of "main factors" in the formulation and process design space for rollercompacted ciprofloxacin hydrochloride immediate-release tablets. AAPS Pharm SciTech. 2012; 13(4):1243-1254.

16. Shivhare M, McCreath G. Practical considerations for DOE implementation in quality by design. BioProcess Technical. 2010, pp. 22-30.

17. Sangshetti J, Deshpande M, Zaheer Z, Shinde D, Arote R. Quality by design approach: regulatory need. Arabian Journal of Chemistry. 2014

18. Rathore A, Winkle H. Quality by design for biopharmaceuticals. Nature Biotechnology. 2009; 27(1):26-34

19. Misar S, Ansari M, Derle D, Bhalerao K. Quality by design: a paradigm for industry. International Journal of Pharmaceutical Sciences Review and Research. 2014; 28(1):67-74.

\section{How to cite this article:}

Patel SR, Dasgupta D. Quality by design approach to protein PEGylation: A review. J App Biol Biotech. 2017; 5 (04): 085-089. 